\title{
Study on cerebral vascular image of spectral domain optical coherence tomography with compressive sensing \\ Ping $\mathrm{Xu}^{1^{*}}$, Zhaoyang $\mathrm{Jin}^{2}$, Yong Yang ${ }^{1}$ \\ 1 College of Life Information Science and Instrument Engineering, Hangzhou Dianzi University, Hangzhou 310027, China \\ pingxu2011@163.com \\ 2 Institute of Information and Control, Hangzhou Dianzi University, \\ Hangzhou 310027, China
}

\section{ABSTRACT}

We propose a novel compressed sensing method to retrieve cerebral vascular image for spectral-domain optical coherence tomography (SD OCT). The compressed sensing method based on 11 norm minimization is applied to reconstruct each A-scan data. The proposed method uses about $25 \%$ of the total data as required in traditional SD OCT to reconstruct the cerebral angiograpy. Therefore this method is favorable for high speed imaging for cerebral angiograpy. It is shown that the proposed method can achieve better performance of axial resolution and higher signal-to-noise ratio (SNR) as compared with the conventional methods.

\section{Keywords}

optical coherence tomography, compressed sensing, cerebral vascular image

\section{Council for Innovative Research}

Peer Review Research Publishing System

Journal: International Journal of Computers \& Technology

Vol 11, No.3

editor@cirworld.com

www.cirworld.com, member.cirworld.com 


\section{INTRODUCTION}

Optical coherence tomography (OCT) is a noninvasive optical imaging technology born in 1991[1]. Due to characteristics of non-invasive, high resolution (less than $10 \mu \mathrm{m}$ ), fast imaging speed, no radiation damage and etc., OCT has become a research hotspot in the field of biological tissue imaging and is considered to be a promising nondestructive in vivo optical imaging tool. OCT has been used widely in modern medical diagnostics, such as eye disease, early diagnosis of tumors, early diagnosis of bone arthritis and .etc. In recent years, due to its better sensitivity and faster imaging speed compared to time domain OCT (TD OCT), spectral-domain OCT (SD OCT) has replaced conventional TD OCT in many medical applications[2].

Applications of SD OCT to cerebral blood flow imaging has become a new hotspot. Cerebrovascular disease is one of the main diseases of mortality and disability, which has always been among the top three disease resulting in human death. Cerebral blood flow information is an important index for the diagnosis of cerebral vascular diseases. High spatial resolution, on the order of micrometers, is necessary to distinguish micrometer-scale vessels (arterioles, venules, capillaries) and individual columns. The resolutions of PET and fMRI are too low to distinguish these components. Due to high spatial and time resolution, SD OCT provides a new technique for the detection of cerebral blood flow. However, the plaques, electromagnetic and scan noise of SD OCT system decreses the imaging quality, which causes cerebrovascular imaging fuzzy and background separation, and affects the detection of cerebral vascular distribution and brain blood flow.

Since 2006, compressed sensing(CS)[3,4] has become a new research hotspot because of its potential ability to significantly reduce the amount of data acquisition in the area of mathematics and signal processing. CS has been applied in medical imaging such magnetic resonance imaging (MRI)[5] and photo-acoustic tomography[6,7]. Until recent years, CS has been introduced in OCT reconstuction[8,9]. SD OCT uses a spectrometer and a linear array CCD to obtain the interferogram. However, to achieve a larger imaging depth with a given axial resolution, it requires more pixels in the array detector to capture the spectral interferogram, which not only limits the imaging speed but also significantly increases the data processing and storage burden[3].

In this thesis, the SD OCT system is established and applied to reconstruct cerebral vascular image for a rat. We propose a novel CS method to reconstruct cerebral vascular image. A random mask is generated for CS reconstruction, which enables random undersampling directly from an original interferogram to get a only a small fractional of points. The total amount of data to be transferred and processed can be dramatically reduced. And the signal-to-noise ratio of the CS reconstructed cerebral vascular image can also be improved. Therefore, the CS is favorable for high speed cerebral angiograpy imaging of SD OCT.

\section{Method and Materials}

\section{System Setup}

Figure 1 depicts the schematic diagram of the established SD OCT setup to reconstruct cerebral vascular image of a rat in vivo. We use a broadband superluminescent diode (SLD 371-HP, Superlum Diodes Ltd.) as light source that has a $\sim 45 \mathrm{~nm}$ effective bandwidth centered at $835 \mathrm{~nm}$. Maximum output power of the source is $12 \mathrm{~mW}$. The light is coupled into the fiberbased Michelson interferometer via a broadband optical circulator (Thorlabs). In the reference arm, the light is delivered onto a stationary mirror. In the sample arm, the light is focused into the sample by an objective lens $(f=75 \mathrm{~mm})$ with a focused spot of $15 \mu \mathrm{m}$. An X-Y galvanometer scanner $(6215 \mathrm{H}$, Cambridge Technology) is used to scan the probe beam transversely over the sample. Light returning from the sample and reference arms are recombined in the fiber coupler and the output interference signal is routed into a custom-built spectrometer via the optical circulator. The spectrometer consists of a $60 \mathrm{~mm}$ focal length achromatic collimating lens (OZ Optics), a 1200 lines/mm transmission grating (Wasatch Photonics), and a 150-mm focal length achromatic focusing lens (Edmund Optics) that images the spectral interference onto a line-scan CCD camera (ATMEL AVIIVA SM2), with a maximum line scan rate of $29 \mathrm{kHz}$. The spectral resolution of the spectrometer with the camera (2048 pixels, with each pixel at $14 \mu \mathrm{m} \times 14 \mu \mathrm{m}$ in size and 12 -bit in digital depth) is $0.0674 \mathrm{~nm}$. The measured axial resolution is $6.8 \mu \mathrm{m}$. The spectral data are transferred to a computer via a high-speed frame grabber board (PCle-1430, National Instruments) for data processing.

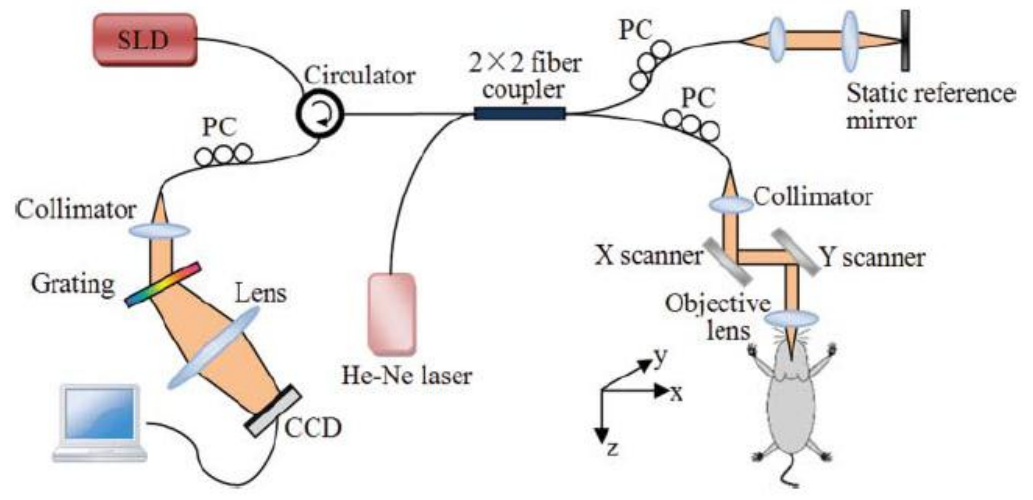

Fig. 1 Schematic of the spectral domain OCT system used to monitor rat's cerebral vascular image. 


\section{Animal Preparation}

An adult Sprague-Dawley rat weighing $250 \pm 20 \mathrm{~g}$ (from Zhejiang Animal Center) is anesthetized with an intraperitoneal injection of urethane $(800 \mathrm{mg} / \mathrm{kg})$. After a rat is anesthetized, a midline scalp incision is made and the parietal bone overlying the sensory cortex is thinned, leaving a thin translucent cranial plate covering an area of $3 \mathrm{~mm} \times 5 \mathrm{~mm}$ centered 2-mm caudally and 2-mm laterally to the bregma. After the animal operation, the rat is fixed in a stereotaxic apparatus, and the data for cerebral vascular image of pial arteries can be achieved in vivo.

\section{SD OCT CS Reconstruction}

The SD OCT CS reconstruction is realized by solving an optimization problem that minimizes the L1 norm of the transformed image:

$$
\text { minimize } \quad a\|\Psi m\|_{1}+\beta T V(m)
$$

$$
\text { s.t. }\left\|F_{u} m-M x\right\|<\varepsilon
$$

Where $\Psi$ is a wavelet transform used to calculate the wavelet sparsity, TV is the total-variation norm used to calculate the finite-difference sparsity, the values of $a$ and $\beta$. provides the relative weighting of the wavelet sparsity and finitedifference sparsity, respectively, $\mathrm{m}$ is the reconstructed image, $M x$ is the measured k-space data, $F_{u}$ is the undersampled Fourier transform, and $\mathcal{E}$. is the threshold parameter used to control the fidelity of the reconstruction relative to the measured k-space data. Minimize both the L1 norm in wavelet domain and the TV norm in total variation domain can promote the sparsity and realize a nonlinear edge-preserving denoising. The constraint $\|F u-M x\|<\varepsilon$ enforces the data consistency.

The constrained convex optimization problem in Eq. (1) was solved by considering its unconstrained form described as follows:

$$
\text { minimize } \quad a\|\psi m\|_{1}+\beta T V(m)+\left\|F_{u} m-M x\right\|
$$

The nonlinear conjugate gradient and backtracking line-search method was applied to solve the unconstrained problem as described in Eq. (2). In our experiment, we use 2D wavelet transform to calculate the wavelet sparsity.

The input interferograms of SD OCT are random subsampled by a mask.For each interferogram, the maximum and minimum data points are firstly selected during each period, and then undersampled at a certain rate in a random manner to further reduces the required data. Fig. 2 gives the interferogram of the 100 th $x$-axis and the red point is the samples(512 points). This undersampled points of each $\mathrm{x}$-axis are then linear interpolated, and all interpolated points are finally introduced into the CS reconstruction. The variable density random sampling with undersampling rate of 0.8 , which samples less where the spectral intensity of interferogram is small and samples more where the spectral intensity is large, is used to select points to further reduce the required data amout to about 25 percents.

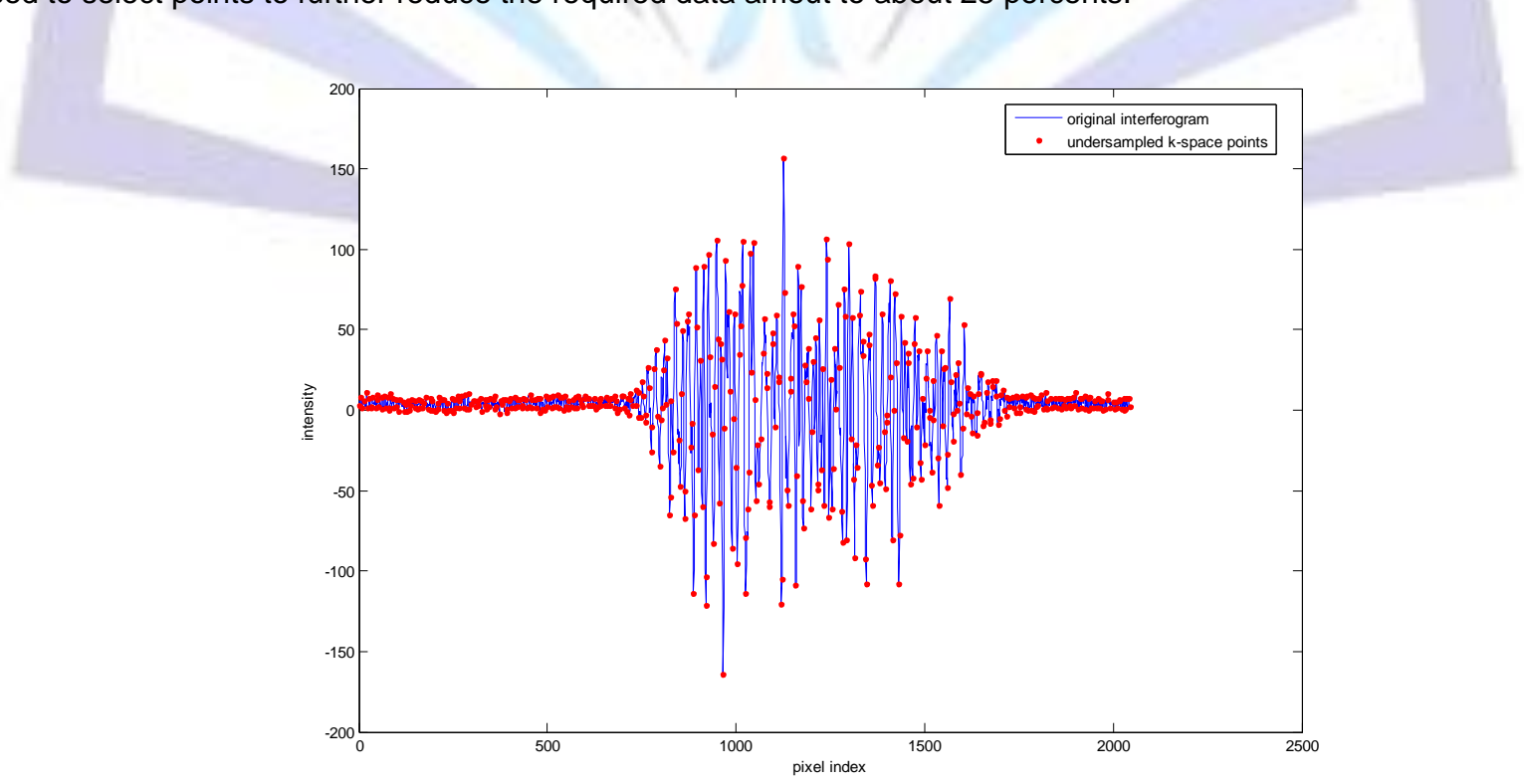

Fig. 2 interferogram of the $100^{\text {th }} x$-axis and $k$-linear sampling points 


\section{Experimental results}

Fig3 shows the structural image of cerebral vascular image with different methods. Fig3.(a) is the original reconstructed image without interpolation. Fig3.(b) shows the reconstructed image with interpolation. Fig3.(c) gives the resultant image using the proposed CS method. The parameters of $a$ and $\beta$ are 0.005 and 0.002 respectively. It is clearly that the axial resolution of the structural image of cerebral vascular image is greatly improved over the original method without interpolation using the proposed CS method. Because the noises of the background of the image are suppressed by the the proposed CS method, the SNR advantage of can be cleatly observed compared to the orignal method without and with interpolation. It is worth mentioning that the the proposed CS method does not show undersampling coherent alising. Fig4 shows another OCT structural images of cerebral vascular image with different methods, which gives similar results. The above results show that the proposed compressed sensing method can achieve high resolution and high SNR structural image of cerebral vascular image with a small amount of data.

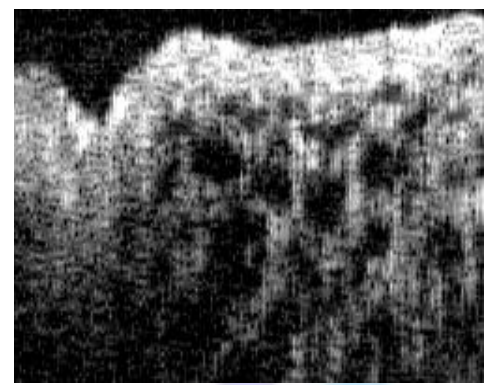

(a) original method without interlation proposed CS method.

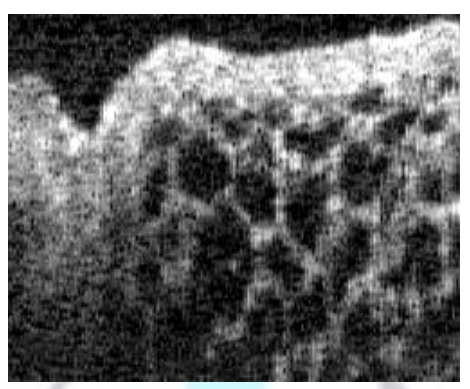

(b) original method with interlation

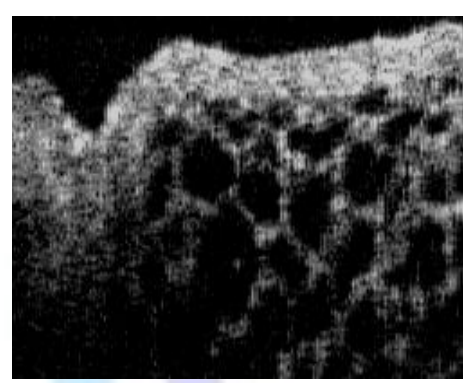

(c) the reconstructed image of the

Fig3. shows another OCT structural images of cerebral vascular image with different methods.
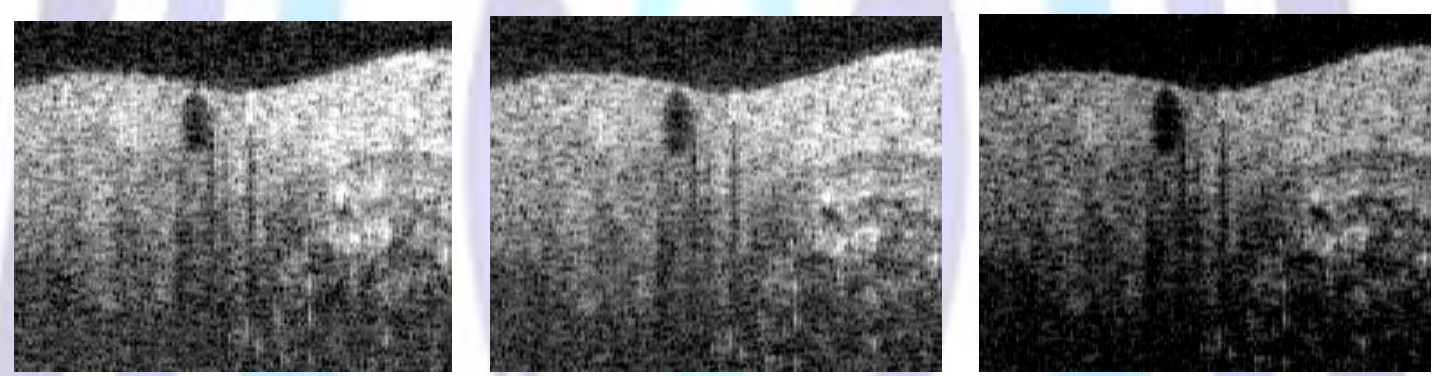

(a) original method without interlation (b) original method with interlation (c) the reconstructed image of the proposed method.

Fig4. shows another OCT structural images of cerebral vascular image with different methods.

\section{Conclusion}

In conclusion, a novel compressed sensing method is developed to reconstruct the structural OCT images of cerebral vascular image. It is shown that the proposed CS method can achieve the even better performance of resolution than the interpolation with about $25 \%$ of the total data and higher SNR, which is favorable for high speed imaging of cerebral vascular image both SD OCT and SS-OCT.

\section{ACKNOWLEDGMENTS}

This research was sponsored by the National Science Foundation of China (NSFC), grants 61205200 and 61372024 , and Zhejiang Provincial Natural Science Foundation of China(ZJNSF), grants LY12F01005 and LY12F03003.

\section{REFERENCES}

[1] D. Huang, E.A. Swanson, C.P. Lin, J.S. Schuman, W.G. Stinson, W. Chang, M.R. Hee, T. Flotte, K. Gregory, C.A. Puliafito, J.G. Fujimoto, "optical coherence tomography”, Science, 254 (1991) 1178.

[2] P.E. Andersen, L. Thrane, H.T. Yura, A. Tycho, T.M. Jørgensen, M.H. Frosz, "Advanced modelling of optical coherence tomography systemsPhys", Med. Biol. 49 (2004) 1307.

[3] E. J. Cand`es, J. K. Romberg, and T. Tao, "Stable signal recovery from incomplete and inaccurate measurements", Communications on Pure and Applied Mathematics, 59, 1207 (2006).

[4] D. L. Donoho, "Compressed sensing" , IEEE Transactions on Information Theory, 52, 1289-1306 (2006). 
[5] M. Lustig, D. Donoho, and J. M. Pauly, "Sparse mri: The application of compressed sensing for rapid mr imaging", Magnetic Resonance in Medicine, 58, 1182-1195 (2007).

[6] J. Provost and F. Lesage, "The application of compressed sensing for photo-acoustic tomography", IEEE transactions on medical imaging, 28, 585-594 (2008).

[7] Z. Guo, C. Li, L. Song, and L. V. Wang, "Compressed sensing in photoacoustic tomography in vivo," Journal of Biomedical Optics 15, 021311 (2010).

[8] Xuan Liu and Jin U. Kang, "Sparse OCT: Optimizing compressed sensing in spectral domain optical coherence tomography”, PMC 2012 May 17.

[9] Ning Zhang, Tiancheng Huo, Chengming Wang, Tianyuan Chen, Jing-gao Zheng, and Ping Xue, "Compressed sensing with linear-in-wavenumber sampling in spectral-domain optical coherence tomography", Optics Letters, August 1, 2012 / Vol. 37, No. 15, 3075-3077.

[10] Wei Chen, Zhaoyang Jin, Feng Liu, Yiping P. Du, "Application of SVD-based sparsity in Compressed Sensing Susceptibility Weighted Imaging", 2012 5th International Conference on BioMedical Engineering and Informatics (BMEI 2012), 311-314.

[11] M. Hong, Y. Yu, H. Wang, F. Liu and S. Crozier, "Compressed sensing MRI with singular value decoposition- based sparsity basis", Phy. Med, Biol. vol. 56, pp.6311-6325, 2011.

\section{Author' biography}

${ }^{1}$ Ping Xu was born in 1978. He received the Ph.D degree from Zhejiang university, Hangzhou, China, in 2006. He has been an ssociate professor of College of Life Information Science \& Instrument Engineering of Hangzhou Dianzi University since 2008. His current research interest include compressive sensing, image compression and biomedical image processing.

${ }^{2}$ Zhaoyang Jin was born in 1975. She received the Ph.D degree from Zhejiang university, Hangzhou, China, in 2011. $\mathrm{He}$ has been an ssociate professor of College of Automation of Hangzhou Dianzi University. Her current research interest include compressive sensing and biomedical image processing.

${ }^{3}$ Yong Yang was born in 1963. He received the Ph.D degree from Zhejiang university, Hangzhou, China, in 2004. He has been an professor of College of Life Information Science \& Instrument Engineering of Hangzhou Dianzi University. His current research interest include biomedical engineer and OCT technology. 